\title{
Formulation of maize- and peanut-based semi-synthetic growth media for the ecophysiological studies of aflatoxigenic Aspergillus flavus in maize and peanut agro-ecosystems
}

\begin{abstract}
In studying the ecophysiology of fungal phytopathogens, several stages are involved (in vitro, greenhouse, in planta). Most in vitro studies extensively utilise the general growth media such as Potato Dextrose Agar and Malt Extract Agar. Although the crop components in these media serve as excellent carbon sources and yield luxuriant growth, they are not naturally contaminated with Aspergillus flavus and thus might result in under- or overestimation of its actual toxigenic potentials. Empirical data on the formulation of semi-synthetic growth medium mimicking the natural crop commonly contaminated by A. flavus for the ecophysiological studies in vitro are scarce. The present work was aimed at investigating the ecophysiology of A. flavus on commercial growth media (PDA, MEA); formulating maize- and peanut-based semisynthetic growth media using two methods of raw material preparation (milling, hot water extraction) at different concentrations $(1,3,5,7,9 \% \mathrm{w} / \mathrm{v})$, and comparing the ecophysiological parameters between commercial and formulated growth media. Growth rates were obtained by computing the hyphal expansion data into $\mathrm{y}=\mathrm{mx}+\mathrm{c}$ equation. AFB1 was quantified using high performance liquid chromatography with fluorescence detector. Formulated media were found to yield significantly higher growth rates when compared to commercial media. However, commercial media yielded significantly higher AFB1 when compared to all formulated media. Between the two formulations, milling yielded significantly higher growth rates and AFB1 when compared to hot water extraction. Although in vitro data cannot directly extrapolate in planta performance, results obtained in the present work can be used to gauge the actual toxigenic potential of A. flavus in maize and peanut agro-ecosystems.
\end{abstract}

Keyword: In vitro; Maize; Peanut; Semi-synthetic; A. flavus; Aflatoxins 
